Response to Commentaries on: Telephone-Based, Clinician-Guided Self-Help Cognitive Cognitive Behavioral Therapy for Depression in Parkinson's Disease (dPD): The Responder Cases of "Alice" and "Carl," and the Nonresponder Cases of "Ethan" and "Gary"

\title{
Lessons Learned: Conducting Cases of Manualized, Telephone-Based, Cognitive Behavioral Treatment for Depression in Parkinson's Disease (dPD)
}

\section{LOGAN DURLAND ${ }^{\mathrm{a}, \mathrm{b}}$}

a Springfield Psychological, Springfield, PA

b Correspondence regarding this article should be sent to: Logan Durland, Springfield Psychological, 1489 Baltimore Pike, Suite 250, Springfield, PA 19064

Email: logan.durland@gmail.com

\begin{abstract}
My current clinical practice has been shifted to a telehealth format for the last three months due to the COVID-19 pandemic, and it seems an apt moment to reexamine my participation in Dr.

Roseanne Dobkin's research on manualized telehealth therapy for depression in Parkinson's disease

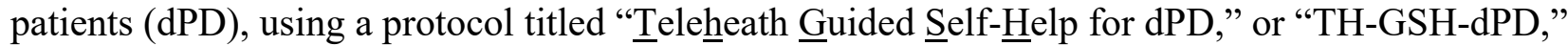
for short (Dobkin et al., 2020). My participation involved, in part, being the therapist in four case studies I have written about with "Alice," "Carl," "Ethan," and "Gary" (Durland, 2020). In these case studies, a subset of those in Dr. Dobkin's group studies, I explored my clinical decisionmaking, seeking insight into how best to flexibly apply the dPD protocol to meet the needs of a heterogeneous clinical population. Here, my aim is to recontextualize and expand on the conclusions of my four case studies, based on my dissertation and conducted over three years ago (Durland, 2017), in light of both my recent experience providing mental health services and the Commentaries on the four case studies so perceptively contributed by Dr. Dobkin and her colleagues (Mann, Miller, St. Hill, \& Dobkin, 2020) and by Liza Pincus (2020). In particular, I will focus first on (a) continuing the analysis of clinical decision-making involved in the case studies described in my earlier article (Duland, 2020); and then on (b) general issues related to the delivery of telehealth treatment.
\end{abstract}

Keywords: telehealth; telemedicine; Parkinson's disease; depression; cognitive-behavioral therapy; motivational interviewing; clinical judgment; case study; clinical case study; pragmatic case studies.

\section{RESPONSE TO THE MANN ET AL. COMMENTARY ON THE FOUR CASE STUDIES: TAILORING THE MANUALIZED TREATMENT TO THE PARTICULAR CLIENT}

\section{Alice and Her Caretaker Husband Bob}

I agree with Mann et al.'s (2020) suggestion that Alice's independence and confidence may have been bolstered by offering her individual treatment sessions. Over the course of our 
work together, Alice made significant progress in taking a more active role in sessions, but she continued to harbor negative beliefs about her functional limitations that might have been effectively challenged through in-session experiences that did not involve her partner.

My recollection is that we discussed the possibility of individual sessions early in treatment, and that I considered it privately later in treatment as Alice began to make clear progress; but I decided not to re-introduce the idea because I was concerned that Alice's progress might be undermined if she found an encounter with her perceived functional limitations without Bob's support and assistance overly demoralizing. Upon further reflection, I think it was a disservice to Alice not to have offered her an opportunity to participate in independent sessions, and in part, I think my hesitation to pursue individual sessions with Alice was rooted in doubts about my ability to handle Alice's potentially negative in-session experience in a constructive way.

Had I carefully framed individual sessions as opportunities to explore her functional capacities and deemphasized pursuit of specific goals, some pressure may have been taken off both Alice and myself, reducing the likelihood of either of us coming away from these sessions feeling as though we had failed may have been mitigated.

Alice's interpretation of her functional limitations led her to experience significant shame, guilt, and frustration. It is possible that despite my efforts to frame these sessions as experiments, Alice may have become preoccupied with negative self-evaluation. To manage this risk, it may have been useful to prioritize reinforcing Alice's openness to individual sessions and signs of effort throughout session, and to frequently assess her in-the-moment experience during session to check for the presence of negative thoughts, feelings and behavioral urges. Instances in which Alice did report such experiences could be treated as opportunities for her to practice applying the cognitive-behavioral therapy model (a) by reflecting on the relationships among her thoughts, feelings and behaviors, and (b) by choosing and implementing an appropriate intervention from prior treatment modules (e.g., cognitive restructuring and/or relaxation strategies) with as little guidance as possible from me. Alice's sense of agency and confidence in her ability to cope with distress could well have been enhanced by encouraging her to take the lead in addressing negative thoughts and feelings in session; and by reinforcing any incremental progress that Alice showed in the direction of independence.

\section{Ethan and His Caretaker Wife, Fay}

With regard to the case of Ethan and Fay, I agree with Mann et al.'s suggestion that individual sessions with each of them may have been helpful. Since Ethan frequently appeared to disengage or defer to Fay in joint sessions, meeting with Ethan individually would have helped me evaluate his functional and cognitive capacities more clearly, and allowed for the treatment protocol to be tailored more precisely to his capacities. I also agree that the criticism and hostility Fay directed at Ethan in session was likely, at least in part, a response to the weight of her caregiving responsibilities, and meeting individually with Fay could have provided her a forum to express these feelings while sparing Ethan's. 
My role in such individual sessions with Fay would have been to normalize and empathize with Fay's caregiving challenges, and frame Ethan's participation in the TH-GSHdPD program as an opportunity to address Fay's caregiver burden as well as Ethan's psychiatric symptoms. Depending on Fay's receptivity, I might have encouraged her to utilize the relaxation skills presented in the TH-GSH-dPD modules to help her cope with her own distress. I like Mann et al.'s recommendation that I could have framed my individual sessions with Ethan as a "break" for Fay. Also it could have been worthwhile to extend this idea by exploring ways to afford Fay regular breaks from her caregiving duties outside of session. One example would have been encouraging Fay to identify family members or friends who might have been able to routinely take temporary responsibility for Ethan to allow Fay time to herself.

\section{Carl and His Caretaker Wife Doris}

Dobkin et al.'s suggestion that I might have been able to facilitate Carl's engagement with treatment by fostering his observation of his in-session experience seems a sound one. This could have created additional opportunities for Carl to gain insight into treatment concepts (like the interdependent relationships among his cognitions, behaviors, and emotions) on his own terms. On the other hand, asking Carl to consider "how those observations might reflect the CBT model," as Mann et al. suggest, would have been a significant challenge.

My struggle to help Carl engage with the TH-GSH-dPD manualized material seemed in part due to my clinical skill deficits and lack of experience. However, I believe these struggles were also the result of Carl's remarkably intense interest in impressionistically relating his subjective experience to me, and his distaste for conceptual abstraction and generalization. Carl found the notion that his experience could be framed in terms of a generalized model to be reductionist and invalidating on its face, and he said as much on a number of occasions. I think an explicit shift of focus towards the treatment model would have been difficult for him, even if his in-session subjective experience were used as a starting point.

In order to minimize the impact of the above challenges, I might have limited the scope of treatment to concepts Carl related to most readily (e.g., the idea of questioning his thoughts). In doing this I could have taken a two-part approach. First, I would have spent significant time instilling these select concepts by repeatedly encouraging Carl to connect the specifics of his experiences to treatment-relevant phenomena, thus reinforcing any incremental improvement in Carl's interest in or ability to participate in this process.

Second, I would have been quite ready to let go of this agenda and join with Carl at moments when he showed he was unable to pursue it. Additionally, Carl's treatment engagement might have been improved by doing more of what ultimately seemed to me most helpful: eschewing the terminology (e.g., "cognition," "behavior," "emotion") presented in the treatment materials in favor of a co-created language for referring to treatment-relevant concepts based on specific, emotionally-salient ideas or examples from Carl's experience and/or our discussions. An example is the "depression genie" idea that emerged in our session about Module 6 on cognitive restructuring : 
Carl shifted his focus to the instance from the past week in which he had experienced catastrophic thoughts in the context of a computer error message, and said at the time he received the message, he believed there was an entity in the computer that was "mocking him." Deciding to go with Carl's framing of the situation, I suggested that we call this entity the "depression genie," and described cognitive restructuring as an effort to prevent the "depression genie" from taking control of his mood and his behavior, which Carl seemed to enjoy. At the end of this discussion, Carl still voiced some doubts about the effectiveness of cognitive restructuring, but said that the concept of the "depression genie" had helped him make sense of cognitive restructuring. We agreed to discuss his concerns further in subsequent sessions (Durland, 2020, p. 34).

\section{Gary}

I agree with Mann et al.'s assessment that Gary's ambivalence at the outset of treatment was significant enough to make therapy focused on ameliorating his depressive symptoms "all but impossible." And that in line with this, what was clinically indicated was postponing the manual-based TH-GSH-dPD treatment, and instead working with Gary's ambivalence towards treatment and difficulty accepting his PD diagnosis.

I also find valuable Mann et al.'s suggestions (2020,p. 113) to work with Gary by taking time to "just talk" as Gary requested, to "negotiate the inclusion of some specific treatmentrelevant topics," and to "elicit more information about Gary's understanding of his depression, its causes and consequences, and his concerns about starting psychotherapy."

Ironically, my early sessions with Gary ended up unfolding along these lines. However, in order for these sessions to have laid the foundation for effective TH-GSH-dPD work, what was crucial was to establish a collaboratively negotiated agenda that explicitly incorporated Gary's present concerns and attitude towards his PD diagnosis, rather than a tug-of-war between his agenda and mine, which was oriented to my following the therapy manual. Helping Gary develop sufficient acceptance of his diagnosis and openness to engaging in psychotherapy likely would have taken multiple sessions. While it is possible that Gary would have been open to

"trying on" the CBT model early in this process, I think it would have been important for me to be willing at times to entirely set aside the TH-GSH-dPD treatment agenda.

As evidence that Gary was at least partially open to psychotherapy, Mann et al. proposed:

Given the time-consuming treatment trial enrollment process, Durland could presume that despite the urgency Gary felt in many areas of his life, he had expended significant effort to access this form of treatment at this time, only to withdraw when it became available (2020, p. 113).

I agree with this point. Additionally, I think Gary entered treatment with vague hopes that the TH-GSH-dPD treatment would directly address his extrapyramidal PD symptoms (e.g., cognitive decline, tremor, and bradykinesia) in addition to his psychiatric symptoms, and I believe his realization that these former symptoms would not be treatment targets in our work together contributed to his disengagement early in therapy. 


\section{RESPONSE TO THE PINCUS COMMENTARY: TELEHEALTH THERAPY IN THE WORLD OF COVID-19 AND BEYOND}

Liza Pincus's (2020) Commentary on my four cases offers useful ideas for extending my discussion of clinical decision-making issues in those cases to telehealth therapy practice in today's world of COVID-19. In particular, she suggests that some of the issues I encountered delivering telehealth therapy over the phone-specifically, (a) difficulty managing tangentiality without the use of body language and visual cues, and (b) coping with potential interference with rapport mediated by connection problems - may be mitigated by use of videoconference technology.

Based on my experience delivering psychotherapy exclusively via videoconference technology in response to the COVID-19 pandemic, I generally agree on this point. I feel I have benefited from access to visual cues and body language that videoconference technology provides.

Based on an informal assessment of patients' perception of rapport and my attunement in session, strikingly approximately $80 \%$ of my patients have stated that they are unaware of any appreciable difference in these domains compared to in-person sessions. On the other hand, my experience has also been that physical connection problems are far more common when conducting teletherapy via videoconference than via the telephone, and in some cases I have found myself in the position of deciding what degree of connection interference during a videoconference call warrants switching to the telephone modality. Generally, my patients have expressed a preference for videoconference sessions, which has complicated this decision when connection difficulties in videoconference sessions are significant.

Another issue relevant to both videoconference telehealth therapy involves variations in transmission speed or lag time that can substantially limit fluidity of dialogue. As a clinician, these situations cause concern about the potential impact of stilted communication on patients' and my sense of connection and engagement. I have not found empirical research addressing this issue, and as stated above, the feedback I have received from my patients does not indicate that they experience this issue as significant. Some scholars suggest that clinicians' concerns impede the adoption of the telehealth treatment modality more than those of patients (Saeed, Bloch and Diamond, 2014). In the absence of empirical evidence, and following discussions with colleagues about their experience with this issue, I have come to the conclusion that continuing to entertain these concerns is more likely to negatively impact the quality of treatment by disturbing my in-session demeanor than the lag time/transmission speed issue itself.

A related challenge involves coping with patients' difficulties utilizing videoconference technology. A substantial minority of my patients are senior citizens, and many have had difficulty using videoconference technology despite significant assistance from friends and family or myself, in some cases necessitating a discussion of whether to shift therapy to the telephone. The significance of these issues for a given patient has been influenced by the availability of a family member or friend to provide technical assistance to the patient at the time of session, which has led to scheduling changes in certain cases. Despite most patients' 
preference for videoconference technology, over the course of the COVID-19 pandemic I have lowered my threshold for suggesting a switch from videoconference to telephone with technologically-naïve patients, since ongoing technological issues often cut into session time. Moreover, I have become increasingly confident that, despite patients' general stated preference for the videoconference modality, at least anecdotally there is not a substantial difference in therapeutic outcome between telephonic and videoconference teletherapy.

Pincus's emphasis on the benefit of teletherapy in eliminating geographical barriers to treatment is well-supported in the literature (Dobkin et al., 2013). Several of the patients I saw in-person prior to the COVID-19 pandemic and who live a significant distance from my office have stated that they would prefer to continue conducting therapy via telehealth even after inperson sessions are possible because they have concluded that telehealth treatment offers significant savings of time (e.g., commute) and money (e.g., tolls and gas), without compromising quality of treatment.

Additionally, Pincus touts the advantages of delivering telehealth treatment via mobile phone rather than other videoconference technology in delivering telehealth treatment due to added portability. I have found this borne out during the pandemic, as several patients who for a variety of reasons were unable to secure privacy within their residence or were in the midst of travelling were able to conduct sessions effectively using their mobile phones.

Pincus points out the crucial role caregiver involvement played in several of the four cases I described, and she cites increased opportunity for caregiver involvement as an advantage of telehealth treatment. In the course of providing telehealth treatment during the COVID-19 pandemic, a number of instances have arisen in which I believe the telehealth treatment modality allowed for the participation of caregivers and family members who would have been unlikely to participate in face-to-face sessions. This is often because telehealth allows caregivers who are in the same location as the patient to participate briefly in a session with minimal disruption to their schedule (e.g., travel). In a related point, caregivers who are not in the same location as the patient can be included in telehealth sessions via the conference call function of telehealth platforms.

Also, telehealth sessions, particularly videoconference sessions in which the patient is at home, can offer valuable information about a patient's everyday environment. One woman with a trauma history presented for a telehealth session in a significantly less agitated state than I was used to during our face-to-face sessions, and, with her cat nestled in her arms, described how her pets give her life meaning and help her cope with distress. She had never talked about her pets in this way, and her relationships with her pets ultimately became the basis for coping strategies that helped her engage with in vivo and imaginal exposures targeting traumatic experiences.

The question of how and when to modify a manualized treatment protocol was a central focus of my four case studies, and Pincus offers useful input on this topic with regard to several of the presented case studies. I agree with Pincus's suggestion that Motivational Interviewing (MI) may have served as an effective precursor to TH-GSH-dPD treatment by helping my client Gary work towards acceptance of his diagnosis by generating greater motivation to engage with treatment. Motivational Interviewing emphasizes "resisting the righting reflex"- defined as "the 
tendency of practitioners to try to actively fix problems in their clients lives, and by so doing, reducing the likelihood of client change" (Rosengren, 2017).

In reviewing my recollections and notes on my sessions with Gary, he clearly struggled to connect with and sometimes actively resisted aspects of the TH-GSH-dPD protocol that may have been experienced as attempts to "fix" his problems (e.g., cognitive restructuring) without adequately exploring and validating his ambivalence about accepting his diagnosis and addressing his psychiatric symptoms. Other treatment adaptations relevant to the case of Gary were addressed above in my response to the commentary by Mann et al.

\section{CONCLUSION}

In reflecting on my case studies of Alice, Carl, Ethan, and Gary - conducted over three years ago - in light of my experience providing telehealth treatment during the COVID-19 pandemic and the thoughtful Commentaries of Mann et al. (2020) and Pincus (2020), two lessons stand out.

First, the importance of clinical flexibility, both in the means of service delivery and in treatment strategy within a given theoretical framework, seems unequivocally clear. The success and failure of the treatment described in the case studies of Alice, Carl, Ethan, and Gary hinged to a substantial extent on the flexibility of my treatment approach, or lack thereof.

Likewise, since completing those case studies over three years ago and in my current experience as a therapist in the time of the COVID-19 pandemic, without being willing to transition to delivering therapy via telehealth technology and troubleshoot related technical and ethical challenges, I would have been unable to properly and effectively serve my current clients.

Secondly, the importance of regular self-assessment and consultation with other professionals as a means of guarding against the deleterious influence of personal insecurities or biases has been underscored for me. My own self-reflection along with feedback from colleagues helped me bring awareness to the possible negative influence of my own concerns about maintaining rapport within a telehealth modality. Dr. Dobkin's clinical supervision of the four cases, described in my case study article (Durland, 2020) and Mann et al.'s Commentary has helped me to recognize that my prioritization of adherence to the research study treatment protocol, while well intentioned, interfered with my ability to individually tailor treatment effectively to Alice, Bob, Ethan, and Gary. I've concluded that it is this ability for self-reflection, done individually and in peer supervision/consultation, that is paramount for best practice and has to remain a major focus in my ongoing clinical work. 


\section{REFERENCES}

Dobkin, R. D., Mann, S. L., Gara, M. A., Interian, A., Rodriguez, K. M., \& Menza, M. (2020, April 21). Telephone-based cognitive behavioral therapy for depression in Parkinson disease. A randomized controlled trial. Neurology, 94 (116). doi: 10.1212/wnl.0000000000009292

Dobkin, R. D., Rubino, J. T., Friedman, J., Allen, L. A., Gara, M. A., \& Menza, M. (2013). Barriers to mental health care utilization in Parkinson's disease. Journal of Geriatric Psychiatry and Neurology, 26(2), 105-116.

Durland, L. (2017). Telephone-based cognitive behavioral therapy for depression in Parkinson's disease: Four pragmatic cases. Unpublished doctoral dissertation, Rutgers University, Piscataway, NJ.

Durland, L. (2020). Telephone-based, clinician-guided self-help cognitive behavioral therapy for depression in Parkinson's disease (dPD): The responder cases of "Alice" and "Carl," and the nonresponder cases of "Ethan" and "Gary." Pragmatic Case Studies in Psychotherapy, 16(1), Article 1, 1-103. Available: http://pcsp.libraries.rutgers.edu/

Mann, S.L., Miller, R., St. Hill, L., \& Dobkin, R.D. (2020). Individualizing evidence-based treatment of neuropsychiatrically complex patients: Process-based targets for change in Parkinson's depression. Pragmatic Case Studies in Psychotherapy, 16(1), Article 2, 104117. Available: http://pcsp.libraries.rutgers.edu/

Pincus, L.E. (2020). Telemental health during a global pandemic: Clinical lessons from guided self-help, telephone therapy case studies. Pragmatic Case Studies in Psychotherapy, 16(1), Article 3, 118-123. Available: http://pcsp.libraries.rutgers.edu/

Rosengren, D. B. (2017). Building motivational interviewing skills: A practitioner workbook. New York: Guilford publications.

Saeed, S. A., Bloch, R. M., \& Diamond, J. M. (2012). Telepsychiatry: Overcoming barriers to implementation. Current Psychiatry, 11(12), 29. 